\title{
Estrutura e diversidade de trechos de Cerrado sensu stricto às margens de rodovias no estado de Minas Gerais
}

\author{
Structure and diversity of Cerrado sensu stricto patches along roadsides in Minas \\ Gerais state
}

\author{
João Paulo Costa ${ }^{\mathrm{I}}$, Lilian Cristina da Silva Santos ${ }^{\mathrm{II}}$, Jovan Martins Rios ${ }^{\mathrm{III}}$, \\ Amanda Wolberg Rodrigues ${ }^{\mathrm{IV}}$, Olavo Custódio Dias Neto ${ }^{\mathrm{V}}$, \\ Jamir Prado-Júnior ${ }^{\mathrm{VI}}$, Vagner Santiago do Vale $\mathrm{VII}$
}

\begin{abstract}
Resumo
Estradas são um dos principais fatores de degradação conhecidos do Cerrado, pois, apesar de sua relevância econômica, causam recortes em extensas massas contínuas de biota natural. De acordo com o Código Florestal Brasileiro, a vegetação às margens de rodovias deve ser considerada como de preservação permanente. Entretanto, escassos são os estudos que visam avaliar o real potencial conservacionista destas áreas. O objetivo do estudo foi avaliar o estágio de conservação do estrato arbóreo do Cerrado localizado nas faixas de proteção das rodovias MG-190, MG-223, BR-365 e BR-352. Foram amostradas 40 parcelas de 50 x 10 m nas margens dessas rodovias e demarcados todos os indivíduos arbóreos vivos com circunferência a $30 \mathrm{~cm}$ do solo maior ou igual $15 \mathrm{~cm}$. Foram calculados os parâmetros fitossociológicos de densidade, dominância e frequência relativa, valor de importância, equabilidade de Pielou e os índices de diversidade de Shannon-Weaver e Simpson. A riqueza foi estimada utilizando os estimadores Jackknife $2 \mathrm{e}$ Bootstrap. Foram amostrados 790 indivíduos pertencentes a 88 espécies e 35 famílias. O índice de diversidade de Shannon-Weaver foi 3,64 nats.ind ${ }^{-1}$, o índice de Simpson foi 0,94 e a equabilidade foi 0,81. As espécies mais comuns no levantamento foram Dalbergia miscolobium, Piptocarpha rotundifolia, Machaerium acutifolium, Ouratea hexasperma e Stenocalyx dysentericus. As famílias com maior número de espécies foram Fabaceae (20 espécies), Myrtaceae (8 espécies) e Bignoniaceae (7 espécies). A riqueza estimada foi de 108 espécies pelo estimador Bootstrap e de 159 pelo estimador Jackknife 2, demonstrando que o número de espécies nas margens das estradas pode ser maior do que a encontrada em alguns Cerrados protegidos. Pode-se inferir que as margens de rodovias conservam um importante montante da flora arbórea do Cerrado, o que preconiza a sua manutenção, dado a atual situação de degradação intensiva do Cerrado.
\end{abstract}

Palavras-chave: Antropização; Conservação; Fitossociologia

\section{Abstract}

Roads are one of the main known degradation factors of the Cerrado because, despite their economic relevance, they cause cuts in extensive continuous masses of natural biota. According to the current legislation, roadside borders should be considered as permanent preservation areas. However, there are few studies that aim to evaluate the real conservationist potential of these protection areas. We sampled 40 plots of $50 \times 10 \mathrm{~m}$, arranged randomly at the edge of highways, and sampled all living tree individuals with circumference at $30 \mathrm{~cm}$ from the ground greater or equal to $15 \mathrm{~cm}$. The phytosociological parameters of relative density, dominance and frequency, importance value, Pielou equability and the diversity indices of Shannon-Weaver and Simpson were calculated. The richness was estimated using Jackknife 2, Bootstrap and Mao Tao indices. We sampled 790 individuals belonging to 88 species and 35 families, with a high diversity index of 3.64 for Shannon-Weaver, 0.94 for Simpson, and 0.81 for equability. The most representative species were Dalbergia miscolobium, Piptocarpha rotundifolia, Machaerium acutifolium, Ouratea hexasperma and Stenocalyx dysentericus. The families with the highest number of species were Fabaceae (20 species), Myrtaceae ( 8 species) and Bignoniaceae ( 7 species). The estimated richness was 108 by the Bootstrap estimator and 159 by the Jacknife 2 estimator, demonstrating that the number of species at the roadsides tends to be greater than those found in some protected Cerrado areas. Based on our results, we can infer that the roadside borders are important to conservation, harboring a great amount of the Cerrado tree flora, which justifies their protection by law and recommends their maintenance, given the current intensive degradation of the Cerrado biome.

Keywords: Disturbance; Conservation; Phytosociology

Biólogo, MSc, Programa de Pós-Graduação stricto sensu em Produção Vegetal, Universidade Estadual de Goiás, Rod. GO 330, Km 241, Anel Viário, s/n, Campus Ipameri, CEP 75780-000, Ipameri (GO), Brasil. joaopaulo mc@hotmail.com (ORCID: 0000-0001-5271-8231)

Bióloga, MSc., Programa de Pós-Graduação stricto sensu em Produção Vegetal, Universidade Estadual de Goiás, Rod. GO 330, Km 241, Anel Viário, s/n, Câmpus Ipameri, CEP 75780-000, Ipameri (GO), Brasil. liliancristina_2011@ hotmail.com (ORCID: 0000-0002-6827-2438)

III Engenheiro Florestal, MSc., Programa de Pós-Graduação stricto sensu em Produção Vegetal, Universidade Estadual de Goiás, Rod. GO 330, Km 241, Anel Viário, s/n, Campus Ipameri, CEP 75780-000, Ipameri (GO), Brasil. jovan.jmr@gmail.com (ORCID: 0000- 0002-76356623)

Iv Bióloga, Fundação Carmelitana Mário Palmério, Av. Brasil Oeste, s/n, Jardim Zenith, CEP 38500-000, Monte Carmelo (MG), Brasil. amanda wbro@hotmail.com (ORCID: 0000-0001-5800-001X)

Biólogo, MSc., Professor Celetista da Fundação Carmelitana Mário Palmério, Av. Brasil Oeste, s/n, Jardim Zenith, CEP 38500-000, Monte Carmelo (MG), Brasil. olavonneto@gmail.com (ORCID: 0000-0002-6828-6784)

vı Biólogo, Dr., Programa de Pós-graduação em Ecologia e Conservação de Recursos Naturais, Universidade Federal de Uberlândia, Campus Umuarama, Av. Ceará, s/n, bloco 2D, sala 26, CEP 38400-902, Uberlândia (MG), Brasil. jamirjunior@yahoo.com.br (ORCID: 0000-0003-1917-1283) VII Biólogo, Dr., Professor Estatutário da Universidade Estadual de Goiás, Rod. GO 330, Km 241, Anel Viário, s/n, Campus Ipameri, CEP 75780-000, Ipameri (GO), Brasil. vagner.vale@ueg.br (ORCID: 0000-0003-2155-9156) 


\section{Introdução}

O Cerrado em sua extensão original ocupava uma área de 2 milhões de quilômetros quadrados, se estendendo através dos estados da Bahia, Goiás, Maranhão, Mato Grosso, Mato Grosso do Sul, Minas Gerais, Paraná, Piauí, São Paulo e Tocantins, além do Distrito Federal, totalizando $24 \%$ do território nacional, sendo assim, o segundo maior bioma brasileiro, atrás apenas da Floresta Amazônica (KLINK; MACHADO, 2005; RIBEIRO; WALTER, 2008). É reconhecido pela sua alta biodiversidade, pois contribui com cerca de $5 \%$ da diversidade de fauna e flora do mundo e com cerca de $1 / 3$ da biodiversidade brasileira e isto se deve em parte ao mosaico de fitofisionomias existentes no Cerrado, estando inclusas formações campestres, savânicas e florestais (ANDRADE; FELFILI; VIOLATTI, 2002; VASCONCELOS, 2012). Por estas razões, o Cerrado é considerado uma das 34 áreas críticas para a conservação (hotspots), conceito que tem por base duas premissas principais: ameaça e endemismo, visto que de suas 12.669 espécies confirmadas, 4.125 são endêmicas (MITTERMEIER et al., 2004; FORZZA et al., 2012).

Apesar de sua reconhecida importância ecológica, até 2008 , cerca de $47,48 \%$ de sua cobertura vegetal havia sido perdida (BRASIL, 2011). O fator que mais contribui para a degradação do Cerrado é o desmatamento desenfreado devido ao avanço da agricultura e da pecuária, urbanização e construção de estradas, gerando a fragmentação de habitat e tornandoos incapazes de proteger sua biodiversidade (MACHADO et al., 2004; TEIXEIRA et al., 2004). Contudo, a construção de estradas é um dos maiores fatores de fragmentação conhecidos atualmente, pois causa recortes em extensas massas contínuas de biota natural (BRASIL, 2007).

As estradas, quando em bom estado de conservação, representam a força motriz do desenvolvimento socioeconômico de um país, gerando ganho de produtividade ao facilitar o acesso ao consumidor, promovendo a competição entre concorrentes e uma consequente redução no preço final dos produtos (MENDES, 2011). Porém, apesar de sua notável importância, a construção de estradas causa diversos impactos ambientais (ecológicos e visuais, na qualidade do ar, impactos sonoros e na qualidade da água), por conta da grande quantidade de gases poluentes liberados pelos motores a combustão, abandono de resíduos nas margens, corte seletivo, perda de diversidade biológica e alterações no solo, o que irá refletir diretamente no ambiente, alterando as diferentes fitofisionomias, inclusive aquelas presentes no bioma Cerrado (SAMPAIO; BRITO, 2009).

De acordo com o novo Código Florestal Brasileiro (Lei № 12.651, de 25 de Maio de 2012), as margens de rodovias devem ser consideradas áreas de preservação permanente na forma de faixas ao longo de sua extensão, garantindo assim proteção à paisagem, a estabilidade geológica, a biodiversidade, o fluxo gênico da fauna e flora, proteger o solo e assegurar o bem-estar das populações humanas. Entretanto, escassos são os estudos que visam avaliar o real potencial conservacionista destas faixas de proteção.

Considerado isto, o presente trabalho teve como objetivo determinar o atual estágio de conservação do estrato arbóreo do Cerrado localizado nas faixas de proteção das rodovias situadas nas regiões do Triângulo Mineiro e Alto Paranaíba em Minas Gerais. Foram feitas análises fitossociológicas, estruturais e comparações com outros estudos em áreas nativas de Cerrado, para que fosse possível verificar: 1) o quanto os impactos antrópicos aos quais estas comunidades estão sujeitas, alteram sua riqueza e estrutura; e, 2) verificar a importância destas comunidades para a conservação das espécies arbóreas existentes nas formações savânicas do Bioma Cerrado. 


\section{Material e métodos}

\section{Local de estudo}

O estudo foi realizado às margens de rodovias, conhecidas como "faixas de proteção", ao longo de quatro rodovias de pista simples localizadas no Triângulo Mineiro e Alto Paranaíba (MG-190, MG-223, BR-365 e BR-352) (Figura 1). Tais faixas são protegidas pela legislação federal, mas a carência de estudos dificulta a compreensão de seu real valor ecológico e conservacionista.

$\mathrm{Na}$ área de estudo, existe um mosaico de fitofisionomias florestais e savânicas localizadas na beira das rodovias. No entanto, este trabalho focou apenas nas fitofisionomias típicas de Cerrado sensu stricto (SS) (RIBEIRO; WALTER, 2008) também conhecidas como savanas arborizadas (IBGE, 2012), não inventariando áreas florestadas. Os cerrados SS foram escolhidos por serem uma fisionomia comum e abrangente na ecorregião de estudo, abrangendo oito estados do Brasil Central, inclusive Minas Gerais (RATTER; BRIDGEWATER; RIBEIRO, 2003) e pelo fato da região estar inserida no Bioma Cerrado, considerado um hotspot de biodiversidade (MITTERMEIER et al., 2004). A pluviosidade média anual da região é de $1.550 \mathrm{~mm}$, distribuídos irregularmente, com um verão quente e chuvoso de outubro a março e um inverno frio e seco de abril a setembro, sendo a temperatura média do ar anual de $22^{\circ} \mathrm{C}$. Na região de estudo predominam essas características climáticas, que se enquadram no tipo Aw (KOTTEK et al., 2006).

\section{Figura 1 - Localização geográfica das parcelas na região do estudo.}

Figure 1 - Geographic location of the plots in the study region.

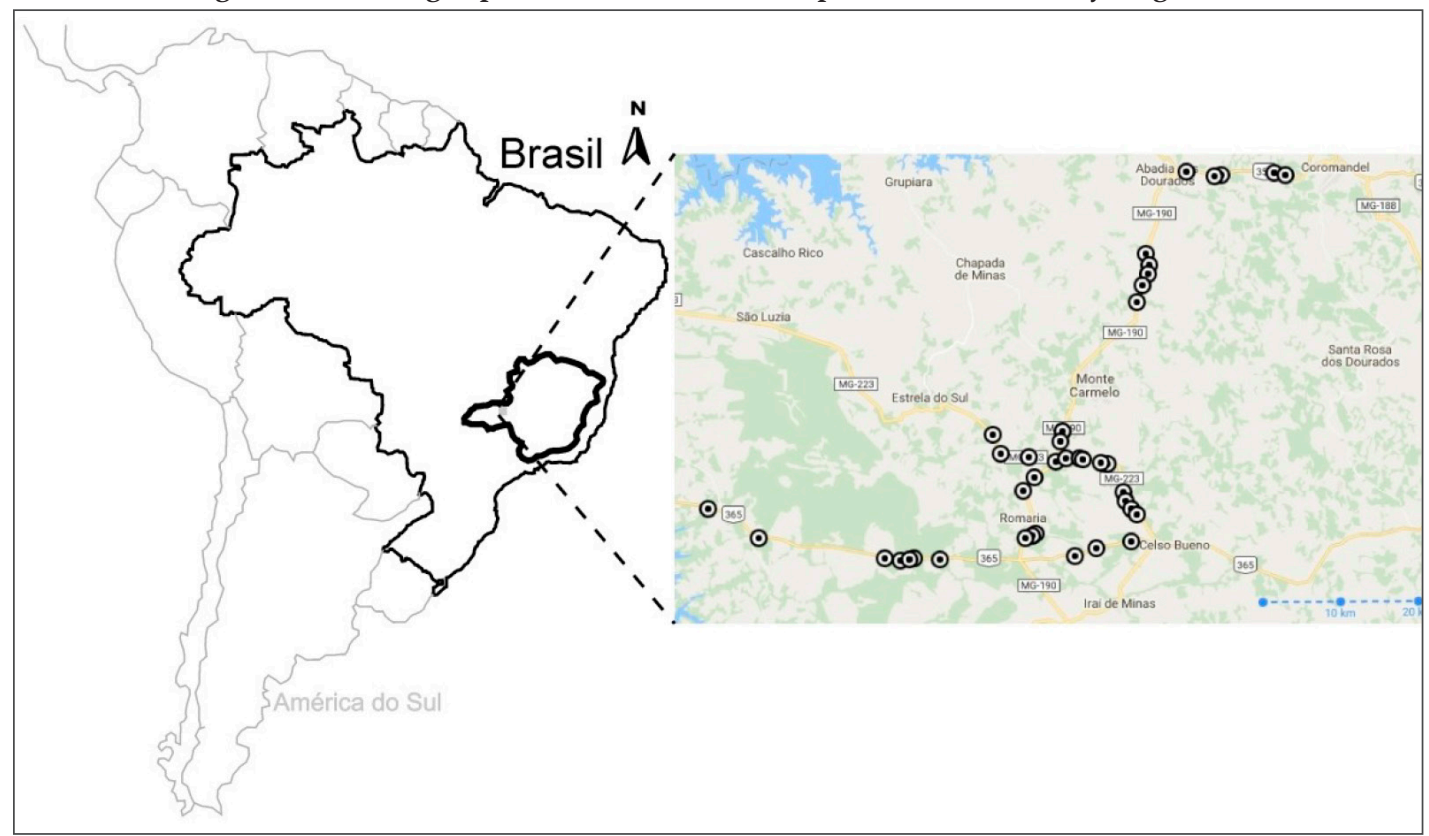

Fonte: Google Earth, 2017)

Source: (Google Earth, 2017)

\section{Amostragem da vegetação}

A coleta de dados foi realizada de março a setembro dos anos de 2015 e 2016. A área de estudo abrangeu 40 parcelas de $50 \mathrm{~m}$ x $10 \mathrm{~m}$ com uma distância média de 18,56 km entre si, dispostas aleatoriamente às margens das rodovias do Triângulo Mineiro e Alto Paranaíba (Figura 1), contabilizando uma área total de $20.000 \mathrm{~m}^{2}$ (2 hectares) e localizadas nas proximidades 
dos municípios de Monte Carmelo, Abadia dos Dourados, Romaria, Iraí de Minas, Uberlândia e Coromandel. Foram demarcados todos os indivíduos arbóreos vivos com circunferência a 30 $\mathrm{cm}$ do solo maior ou igual a $15 \mathrm{~cm}\left(\mathrm{C}_{30} \geq 15 \mathrm{~cm}\right)$, conforme método proposto pela Rede de Parcelas Permanentes dos Biomas Cerrado e Pantanal (FELFILI; CARVALHO; HAIDAR, 2005). As espécies foram identificadas no campo ou por meio de coleta de material botânico.

Além da $\mathrm{C}_{30}$, cada indivíduo teve sua altura medida com uma vara graduada quando inferiores a $2 \mathrm{~m}$ e com uso de clinômetro quando superiores a $2 \mathrm{~m}$. A classificação das famílias foi feita de acordo com o sistema Angiosperm Phylogeny Group IV (BYNG et al., 2016) e os nomes específicos foram atualizados utilizando o Tropicos ${ }^{\circledR}$ (TROPICOS.ORG, 2016).

\section{Fitossociologia das parcelas às margens de rodovias}

Foram calculados os parâmetros fitossociológicos de densidade, dominância e frequência relativas e o valor de importância (VI) (MATTEUCCI; COLMA, 1982). Para a avaliação da diversidade alfa, foram utilizados os índices de diversidade de Shannon (H') e de Simpson (D'), e para aferição da equabilidade foi utilizado o índice de Pielou (J') (BROWER; ZAR, 1984). A diversidade alfa incorpora tanto o número de espécies quanto a abundância relativa de cada uma na comunidade e, quanto maior for a diversidade, mais complexa será a estrutura da comunidade local (RAD; MANTHEY; MATAJI, 2009). A equitabilidade é um valor referência ao quão uniforme é o número de indivíduos de cada espécie na área e, valores menores indicam que poucas espécies dominam o local (RAD; MANTHEY; MATAJI, 2009).

\section{Estimadores de riqueza e curvas do coletor}

A riqueza foi estimada através de 1.000 randomizações utilizando os estimadores Jackknife 2, Bootstrap e Mao Tau, através do programa Estimates 8.2 (COLWELL, 2005). Sendo Mao Tau utilizado para construir a curva do coletor, pois o mesmo indica a riqueza amostrada da área e os demais estimadores de riqueza foram utilizados para estimar a riqueza esperada, por serem considerados bons para esta finalidade (KREBS, 2000).

\section{Comparação com cerrados pouco impactados}

Para fins de comparação, os resultados do atual estudo foram confrontados com os resultados de 18 trabalhos publicados em outros Cerrados localizados em parques/reservas legais e/ ou Cerrados sem grandes perturbações antrópicas. Todos os estudos apresentaram metodologia com critério de inclusão de $15 \mathrm{~cm}$ de circunferência ou $5 \mathrm{~cm}$ de diâmetro (salvo duas exceções, cuja circunferência mínima foi de $10 \mathrm{~cm}$ ), mensurados ou na base do solo ou a $30 \mathrm{~cm}$ de altura do solo. Todos os dados foram padronizados por hectare. Para verificar as relações entre as variáveis estruturais foram realizadas análises de correlações de Pearson entre as variáveis: número de espécies (riqueza), densidade, área basal e índice de diversidade de Shannon para todos os estudos já publicados, inserindo os dados deste estudo.

\section{Resultados e discussão}

\section{Fitossociologia das parcelas às margens de rodovias}

Nas 40 parcelas situadas às margens de rodovias, foram amostrados 790 indivíduos (395,00/ha), pertencentes a 88 espécies e 35 famílias (Tabela 1). As espécies mais comuns foram Dalbergia miscolobium Benth.(155 indivíduos), Piptocarpha rotundifolia (Less.) Baker (61 indivíduos), Machaerium acutifolium Vogel (31 indivíduos), Ouratea hexasperma (A. St.-Hil.) Baill. (31 indivíduos), Stenocalyx dysentericus (DC.) O. Berg (24 indivíduos) e Qualea grandiflora Mart. (22 indivíduos). O 
índice de diversidade de Shannon foi de 3,64 nats.ind ${ }^{-1}$, Simpson de 0,94 e a equabilidade de Pielou de 0,81 , valores normalmente encontrados em estudos conduzidos no Cerrado.

As famílias com maior número de espécies foram Fabaceae (20 espécies), Myrtaceae (8 espécies) e Bignoniaceae (7 espécies). As espécies da família Fabaceae possuem um atributo ecológico muito importante, com vários de seus representantes apresentando simbiose com bactérias do gênero Rhizobium, o que possibilita a fixação biológica do nitrogênio atmosférico, conferindo a esta família uma vantagem em relação às demais em condições de baixa fertilidade do solo (SILVEIRA; COSTA; FELFILI, 2009), e sendo a família mais representativa do Cerrado (FRANÇOSO; HAIDAR; MACHADO, 2016). A família Myrtaceae se encontra distribuída por todo o mundo, mas é encontrada principalmente nos países de clima tropical e subtropical, e que neste estudo é representada principalmente pela espécie Stenocalyx dysentericus, que possui distribuição bastante ampla nos estados de Minas Gerais, Goiás e Bahia e apresenta características que confere vantagens à mesma sobre as demais espécies, como súber espesso e fruto carnoso utilizado como alimento pela fauna, o que facilita sua dispersão (FRANZON et al., 2009; PAIVA et al., 2009).

Dentre as espécies, destaca-se Dalbergia miscolobium, que foi responsável por 19,6\% de todos os indivíduos amostrados, com um valor de importância de 17,62\% e apresentando os maiores valores em todos os outros parâmetros avaliados (Tabela 1). Em contrapartida, 38 espécies apresentaram densidade menor ou igual a 1 individuo por hectare, podendo ser ecologicamente consideradas es pécies pouco comuns e/ou raras nas margens de rodovias estudadas (KAGEYAMA; GANDARA; SOUZA, 1998). As estradas promovem a fragmentação de habitat, que é uma das principais causas da deterioração do sucesso reprodutivo das plantas podendo reduzir sua área de ocorrência, sua população, e até mesmo, levar a extinção local (GIRÃO et al., 2007).

Entre as principais espécies desta comunidade, apenas uma teve ocorrência em mais de $50 \%$ das parcelas amostradas no estudo. Dalbergia miscolobium ocorreu em 72,5\% das parcelas, já Ouratea hexasperma e Piptocarpha rotundifolia ocorreram em 40\%, Machaerium acutifolium em $27,5 \%$, Qualea grandiflora em $25 \%$ e Stenocalyx dysentericus em $22,5 \%$ das parcelas do presente estudo. Tal dado indica que apesar de figurarem como as principais espécies desta comunidade, sua presença se deu de modo concentrado em um pequeno número de parcelas, de maneira semelhante a que ocorre em áreas nativas, sendo a distribuição agregada ou em mosaicos ao longo da paisagem (FELFILI; FELFILI, 2001).

Os principais impactos observados nas margens de rodovias são as frequentes queimadas (anuais/bianuais) e vias rurais utilizadas para o trânsito de máquinas agrícolas, dispostas paralelamente à pista de rolamento. Desta maneira a vegetação existente nas margens da rodovia se dispõe em faixas estreitas entre a rodovia asfaltada e a via rural paralela, situação que funciona como uma borda dupla em ambos os lados da comunidade arbórea, o que provavelmente dificulta o estabelecimento de espécies arbóreas, pois potencializa a ação do vento, luminosidade, redução de umidade no solo, que são importantes causadores de mortalidade de espécies arbóreas (LAURANCE; VASCONCELOS, 2009). Os impactos desta situação e do fogo constante parecem possuir efeitos sobre a estrutura das comunidades arbóreas, sendo que o primeiro ocorre anualmente e o segundo é permanente, visto que o solo fica compactado devido à passagem de carros e maquinários agrícolas. Parcelas dispostas nestas estradas apresentaram baixa densidade e riqueza e quase todas também apresentaram sinais de ocorrência de incêndio nas árvores ou gramíneas remanescentes. Como as estradas rurais não atuam na seleção de espécies, por ser um impacto aleatório e devastador, o foco das discussões será o efeito do fogo.

Dalbergia miscolobium tem uma distribuição ampla pelo território brasileiro, compreendendo desde o estado do Piauí até o Paraná. Dalbergia miscolobium possui alta capacidade de nodulação por Rhizobium, o que facilita sua presença em solos com baixa fertilidade (MASSI, 2014). Possui ainda alta capacidade de rebrota pós-fogo e capacidade de acúmulo de reservas nutricionais para sobrevivência, dado estas características, é uma espécie indicada para programas de recuperação de áreas degradadas (BRAZ; KANEGAE; FRANCO, 2000; POTT; POTT, 2002).

A segunda espécie com maior número de indivíduos no estudo, Piptocarpha rotundifolia, 
possui alta capacidade de recuperação da parte vegetativa aérea e ocorrência de floração pós-evento de queimada, o que justifica sua ocorrência nas áreas amostradas, sendo também uma alternativa para recuperação de áreas degradadas (SARTORELLI et al., 2007). Outra espécie com significativa representatividade na área do estudo foi Machaerium acutifolium, outra espécie da família Fabaceae, que além de contar com a fixação biológica de nitrogênio, possui alta capacidade de adaptação em áreas em processo de recuperação (ROSA et al., 2014). Ouratea hexasperma, possui grande capacidade de rebrota pós-fogo (VALE; LOPES, 2007).

Foi observada também, em algumas parcelas, uma presença massiva de espécies do gênero Pinus sp., mais precisamente em áreas adjacentes ou próximas a locais em que existem ou existiam plantações de Pinus para fins comerciais. Diversos estudos já foram publicados demonstrando o potencial de invasão desta espécie exótica, devido à mesma apresentar um rápido crescimento, facilidade de propagação e alta viabilidade de plântulas, sendo inclusive já considerada como um problema ecológico em alguns locais, existindo até legislações que visam monitorar o plantio e o uso da espécie (BOURSCHEID; REIS, 2011; FALLEIROS; ZENNI; ZILLER, 2011; MIASHIKE, 2015).

Entretanto, para a maioria das espécies, os eventos constantes de queima parecem ser prejudiciais em longo prazo, visto que muitas apresentaram poucos indivíduos. Por se situar em uma área de intenso fluxo de automóveis e muitas vezes estarem conjugadas a áreas de lavouras ou pastagem, nota-se uma frequência intensa e regular de incêndios nessas comunidades, seja por finalidade de limpeza ou com viés criminoso. A frequência e intensidade do fogo se revela um fator determinante para as elevadas taxas de mortalidade em campo sujo e no Cerrado sensu stricto (Cerrado SS) após queimadas prescritas (FIEDLER et al., 2004). Quanto maior for a frequência do fogo na área queimada, maior sua influência na estabilidade do ambiente (VASCONCELOS; ARAÚJO; BRUNA, 2014), tornando-o instável. Uma vez que a queima ocorra em curtos espaços de tempo, as rebrotações podem não apresentar cascas espessas o suficiente ou os indivíduos não atingem a altura crítica para o escape do efeito direto do fogo (LOPES; VALE; SCHIAVINI, 2009).

Tabela 1 - Fitossociologia de cerrados em beira de estradas (MG-190, MG-223, BR-352 e BR-365) no Triângulo Mineiro e Alto Paranaíba - MG . Ni = número de indivíduos amostrados, $\mathrm{DeR}$ = densidade relativa, $\mathrm{FrR}=$ frequência relativa, $\mathrm{DoR}=$ dominância relativa, $\mathrm{VI} \%$ = valor de importância em porcentagem.

Table 1 - Phytosociology of Cerrado areas along the roadsides (MG-190, MG-223, BR-352 and BR-365) in Triângulo Mineiro region and Alto Paranaíba, MG state. $\mathrm{Ni}=$ number of individuals sampled, DeR = relative density, FrR = relative frequency, DoR = relative dominance, $\mathrm{VI} \%=$ importance value in percentage.

\begin{tabular}{|c|c|c|c|c|c|c|}
\hline Espécies & Família & $\mathrm{Ni}$ & DeR & FrR & DoR & VI \% \\
\hline Dalbergia miscolobium Benth. & Fabaceae & 155 & 19.62 & 8.33 & 24.1 & 17.35 \\
\hline Piptocarpha rotundifolia (Less.) Baker & Asteraceae & 61 & 7.72 & 4.6 & 7.37 & 6.56 \\
\hline Machaerium acutifolium Vogel & Fabaceae & 31 & 3.92 & 3.16 & 3.81 & 3.63 \\
\hline Ouratea hexasperma (A. St.-Hil.) Baill. & Ochnaceae & 31 & 3.92 & 4.6 & 1.91 & 3.48 \\
\hline Stenocalyx dysentericus (DC.) O. Berg & Myrtaceae & 24 & 3.04 & 2.59 & 2.22 & 2.61 \\
\hline Qualea grandiflora Mart. & Vochysiaceae & 22 & 2.78 & 2.87 & 3.41 & 3.02 \\
\hline Stryphnodendron adstringens (Mart.) Coville & Fabaceae & 22 & 2.78 & 3.45 & 2.39 & 2.87 \\
\hline Erythroxylum suberosum A. St.-Hil. & Erythroxylaceae & 19 & 2.41 & 2.59 & 0.97 & 1.99 \\
\hline
\end{tabular}


Tabela 1 - Continua...

Table 1 - Continuation...

\begin{tabular}{|c|c|c|c|c|c|c|}
\hline Espécies & Família & Ni & DeR & FrR & DoR & VI \% \\
\hline Bowdichia virgilioides Kunth & Fabaceae & 18 & 2.28 & 2.01 & 2.36 & 2.22 \\
\hline Luehea grandiflora Mart. & Malvaceae & 18 & 2.28 & 0.29 & 1.54 & 1.37 \\
\hline Machaerium opacum Vogel & Fabaceae & 18 & 2.28 & 2.3 & 1.89 & 2.16 \\
\hline Leptolobium dasycarpum Vogel & Fabaceae & 17 & 2.15 & 2.3 & 0.9 & 1.78 \\
\hline Aspidosperma macrocarpon Mart. & Apocynaceae & 16 & 2.03 & 2.3 & 0.94 & 1.76 \\
\hline Connarus suberosus Planch. & Connaraceae & 16 & 2.03 & 2.3 & 1.33 & 1.89 \\
\hline Kielmeyera coriacea Mart. \& Zucc. & Calophyllaceae & 15 & 1.9 & 3.45 & 1.18 & 2.18 \\
\hline Schefflera macrocarpa (Cham. \& Schltdl.) Frodin & Araliaceae & 15 & 1.9 & 2.87 & 1.97 & 2.25 \\
\hline Hymenaea stigonocarpa Mart. ex Hayne & Fabaceae & 14 & 1.77 & 2.59 & 2.94 & 2.43 \\
\hline Leptolobium elegans Vogel & Fabaceae & 14 & 1.77 & 1.15 & 1.66 & 1.53 \\
\hline Handroanthus ochraceus (Cham.) Mattos & Bignoniaceae & 13 & 1.65 & 2.59 & 1.09 & 1.77 \\
\hline Solanum lycocarpum A. St.-Hil. & Solanaceae & 13 & 1.65 & 3.16 & 0.71 & 1.84 \\
\hline Brosimum gaudichaudii Trécul & Moraceae & 12 & 1.52 & 2.3 & 1.28 & 1.7 \\
\hline Erythroxylum deciduum A. St.-Hil. & Erythroxylaceae & 11 & 1.39 & 0.86 & 0.55 & 0.94 \\
\hline Platypodium elegans Vogel & Fabaceae & 11 & 1.39 & 1.15 & 1.63 & 1.39 \\
\hline Pinus sp. & Pinaceae & 11 & 1.40 & 1.15 & 8.43 & 3.65 \\
\hline Annona crassiflora Mart. & Annonaceae & 9 & 1.14 & 1.44 & 0.72 & 1.1 \\
\hline Vatairea macrocarpa (Benth.) Ducke & Fabaceae & 9 & 1.14 & 0.57 & 1.53 & 1.08 \\
\hline Myrsine guianensis (Aubl.) Kuntze & Primulaceae & 8 & 1.01 & 0.57 & 0.51 & 0.7 \\
\hline Qualea parviflora Mart. & Vochysiaceae & 8 & 1.01 & 1.44 & 0.35 & 0.93 \\
\hline Aspidosperma tomentosum Mart. & Apocynaceae & 7 & 0.89 & 1.44 & 0.79 & 1.04 \\
\hline Matayba guianensis Aubl. & Sapindaceae & 7 & 0.89 & 0.86 & 0.74 & 0.83 \\
\hline Aspidosperma subincanum Mart. ex A. DC. & Apocynaceae & 6 & 0.76 & 0.57 & 0.55 & 0.63 \\
\hline Schefflera morototoni (Aubl.) Maguire et al. & Araliaceae & 6 & 0.76 & 0.29 & 0.22 & 0.42 \\
\hline Diospyros hispida A. DC. & Ebenaceae & 6 & 0.76 & 0.86 & 1.18 & 0.93 \\
\hline Hymenaea courbaril L. & Fabaceae & 6 & 0.76 & 0.86 & 0.56 & 0.73 \\
\hline Aegiphila verticillata Vell. & Lamiaceae & 5 & 0.63 & 1.15 & 0.3 & 0.69 \\
\hline Astronium graveolens Jacq. & Anacardiaceae & 5 & 0.63 & 0.29 & 0.11 & 0.34 \\
\hline Byrsonima verbascifolia (L.) DC. & Malpighiaceae & 5 & 0.63 & 0.86 & 0.65 & 0.71 \\
\hline Curatella americana $\mathrm{L}$. & Dilleniaceae & 5 & 0.63 & 0.57 & 0.8 & 0.67 \\
\hline Diospyros burchellii Hiern & Ebenaceae & 5 & 0.63 & 1.44 & 0.28 & 0.78 \\
\hline Himatanthus obovatus (Müll. Arg.) Woodson & Apocynaceae & 5 & 0.63 & 0.86 & 0.64 & 0.71 \\
\hline
\end{tabular}


Tabela 1 - Continua...

Table 1 - Continuation...

\begin{tabular}{|c|c|c|c|c|c|c|}
\hline Espécies & Família & $\mathrm{Ni}$ & DeR & FrR & DoR & VI \% \\
\hline Styrax ferrugineus Nees \& Mart. & Styracaceae & 5 & 0.63 & 1.15 & 1.75 & 1.18 \\
\hline Andira paniculata Benth. & Fabaceae & 4 & 0.51 & 0.57 & 0.3 & 0.46 \\
\hline Byrsonima coccolobifolia Kunth & Malpighiaceae & 4 & 0.51 & 0.86 & 0.39 & 0.58 \\
\hline Caryocar brasiliense A. St.-Hil. & Caryocaraceae & 4 & 0.51 & 0.86 & 1.17 & 0.85 \\
\hline Tocoyena formosa (Cham. \& Schltdl.) K. Schum. & Rubiaceae & 4 & 0.51 & 0.29 & 0.94 & 0.58 \\
\hline Davilla elliptica A. St.-Hil. & Dilleniaceae & 3 & 0.38 & 0.57 & 0.05 & 0.33 \\
\hline Eriotheca candolleana (K. Schum.) A. Robyns & Malvaceae & 3 & 0.38 & 0.86 & 1.56 & 0.93 \\
\hline Guapira noxia (Netto) Lundell & Nyctaginaceae & 3 & 0.38 & 0.86 & 0.29 & 0.51 \\
\hline Handroanthus vellosoi (Toledo) Mattos & Bignoniaceae & 3 & 0.38 & 0.57 & 0.36 & 0.44 \\
\hline Tachigali aurea Tul. & Fabaceae & 3 & 0.38 & 0.86 & 0.07 & 0.44 \\
\hline Tachigali vulgaris L.F. Gomes da Silva \& H.C. Lima & Fabaceae & 3 & 0.38 & 0.86 & 0.06 & 0.43 \\
\hline Terminalia argentea Mart. & Combretaceae & 3 & 0.38 & 0.29 & 1.63 & 0.76 \\
\hline Vochysia rufa Mart. & Vochysiaceae & 3 & 0.38 & 0.86 & 0.44 & 0.56 \\
\hline Myrcia tomentosa (Aubl.) DC. & Myrtaceae & 2 & 0.26 & 0.58 & 0.16 & 0.33 \\
\hline Myrcia variabilis DC. & Myrtaceae & 2 & 0.26 & 0.58 & 0.08 & 0.3 \\
\hline Anadenanthera colubrina (Vell.) Brenan & Fabaceae & 2 & 0.25 & 0.29 & 0.14 & 0.23 \\
\hline Annona coriacea Mart. & Annonaceae & 2 & 0.25 & 0.57 & 0.03 & 0.29 \\
\hline Couepia grandiflora (Mart. \& Zucc.) Benth. ex Hook. f. & Chrysobalanaceae & 2 & 0.25 & 0.57 & 0.18 & 0.34 \\
\hline Cybistax antisyphilitica (Mart.) Mart. & Bignoniaceae & 2 & 0.25 & 0.29 & 0.21 & 0.25 \\
\hline Handroanthus chrysotrichus (Mart. ex A. DC.) Mattos & Bignoniaceae & 2 & 0.25 & 0.57 & 0.44 & 0.42 \\
\hline Licania apetala (E. Mey.) Fritsch & Chrysobalanaceae & 2 & 0.25 & 0.29 & 0.04 & 0.19 \\
\hline Miconia albicans (Sw.) Steud. & Melastomataceae & 2 & 0.25 & 0.29 & 0.19 & 0.24 \\
\hline Ouratea spectabilis (Mart. ex Engl.) Engl. & Ochnaceae & 2 & 0.25 & 0.29 & 0.04 & 0.19 \\
\hline Strychnos pseudoquina A. St.-Hil. & Loganiaceae & 2 & 0.25 & 0.29 & 0.22 & 0.25 \\
\hline Anacardium occidentale L. & Anacardiaceae & 1 & 0.13 & 0.29 & 0.11 & 0.17 \\
\hline Plenckia populnea Reissek & Celastraceae & 1 & 0.13 & 0.29 & 0.04 & 0.15 \\
\hline Blepharocalyx salicifolius (Kunth) O.Berg & Myrtaceae & 1 & 0.13 & 0.29 & 0.1 & 0.17 \\
\hline Byrsonima pachyphylla A. Juss. & Malpighiaceae & 1 & 0.13 & 0.29 & 0.02 & 0.14 \\
\hline Callichlamys latifolia (Rich.) K. Schum. & Bignoniaceae & 1 & 0.13 & 0.29 & 0.1 & 0.17 \\
\hline Campomanesia grandiflora (Aubl.) Sagot & Myrtaceae & 1 & 0.13 & 0.29 & 0.11 & 0.17 \\
\hline Casearia sylvestris Sw. & Salicaceae & 1 & 0.13 & 0.29 & 0.04 & 0.15 \\
\hline
\end{tabular}


Tabela 1 - Conclusão...

Table 1 - Conclusion...

\begin{tabular}{|c|c|c|c|c|c|c|}
\hline Espécies & Família & $\mathbf{N i}$ & DeR & FrR & DoR & VI \% \\
\hline Cedrela fissilis Vell. & Meliaceae & 1 & 0.13 & 0.29 & 0.12 & 0.18 \\
\hline Ceiba speciosa (A. St.-Hil.) Ravenna & Malvaceae & 1 & 0.13 & 0.29 & 0.11 & 0.17 \\
\hline Dimorphandra mollis Benth. & Fabaceae & 1 & 0.13 & 0.29 & 0.03 & 0.15 \\
\hline Handroanthus impetiginosus (Mart. ex DC.) Mattos & Bignoniaceae & 1 & 0.13 & 0.29 & 0.03 & 0.15 \\
\hline Heteropterys byrsonimifolia A. Juss. & Malpighiaceae & 1 & 0.13 & 0.29 & 0.02 & 0.14 \\
\hline Machaerium aculeatum (Vell.) Stellfeld & Fabaceae & 1 & 0.13 & 0.29 & 0.07 & 0.16 \\
\hline Myrcia splendens (Sw.) DC. & Myrtaceae & 1 & 0.13 & 0.29 & 0.05 & 0.15 \\
\hline Myrsine umbellata Mart. & Primulaceae & 1 & 0.13 & 0.29 & 0.05 & 0.15 \\
\hline Plathymenia reticulata Benth. & Fabaceae & 1 & 0.13 & 0.29 & 0.06 & 0.16 \\
\hline Pouteria ramiflora (Mart.) Radlk. & Sapotaceae & 1 & 0.13 & 0.29 & 0.36 & 0.26 \\
\hline Rourea induta Planch. & Connaraceae & 1 & 0.13 & 0.29 & 0.18 & 0.2 \\
\hline Salvertia convallariodora A. St.-Hil. & Vochysiaceae & 1 & 0.13 & 0.29 & 0.02 & 0.14 \\
\hline Tachigali aurea Tul. & Fabaceae & 1 & 0.13 & 0.29 & 0.02 & 0.14 \\
\hline Styrax pohlii A. DC. & Styracaceae & 1 & 0.13 & 0.29 & 0.32 & 0.24 \\
\hline Tabebuia aurea (Silva Manso) Benth. \& Hook. f. ex S. & Bignoniaceae & 1 & 0.13 & 0.29 & 0.26 & 0.22 \\
\hline Tachigali melinonii (Harms) Zarucchi \& Herend. & Fabaceae & 1 & 0.13 & 0.29 & 0.51 & 0.31 \\
\hline Vochysia elliptica Mart. & Vochysiaceae & 1 & 0.13 & 0.29 & 0.02 & 0.15 \\
\hline Total & & 790 & 100 & 100 & 100 & 100 \\
\hline
\end{tabular}

Fonte: Autores (2017)

\section{Estimadores de riqueza e curvas do coletor}

Pode-se notar, ao analisar a curva espécie-área (Figura 2), que não foi atingida a estabilidade de espécies para a amostragem nas comunidades situadas em beira de estrada. A curva não obteve estabilidade em nenhum dos estimadores, indicando que a riqueza provavelmente será bem maior caso sejam feitas novas amostragens. Geralmente, para parcelas disjuntas não ocorre estabilização da curva espécie-área (SCHILLING; BATISTA, 2008), e como as parcelas apresentam grande distanciamento espacial (a média de distância entre as parcelas é de 18,56 $\mathrm{Km}$ ) este comportamento já era previsto. Como as rodovias cortam todo o Brasil Central, esperase que a curva não estabilize rapidamente, mesmo que mais parcelas sejam amostradas (desde que se mantenha a mesma disjunção e distanciamento atuais). O estimador de riqueza Bootstrap (mais conservador) sugeriu uma riqueza de 108 espécies e o estimador Jackknife de segunda ordem (menos conservador) sugeriu uma riqueza de 159 espécies, observado isso e tomando-se como base o estimador de riqueza Mao Tau (riqueza amostrada), foi indicada a presença de mais 17 e 68 espécies nestes cerrados antropizados, respectivamente. Tal dado mostra que apenas mantendo estas comunidades, estaremos preservando de 11,9 a 17,5\% das 909 espécies arbóreas presentes no Cerrado (FRANÇOSO; HAIDAR; MACHADO, 2016). 
Figura 2 - Número cumulativo de espécies baseados em estimadores de riqueza em Cerrados localizados nas margens de rodovias (MG-190, MG223, BR352 e BR-365) do Triângulo Mineiro e Alto Paranaíba, MG. Linha continua = riqueza observada (Mao Tau), linha traço-ponto: riqueza estimada Jackknife de segunda ordem e linhas tracejadas: riqueza estimada Bootstrap.

Figure 2 - Cumulative number of species based on richness estimators in the Cerrados located at roadsides (MG state-190, MG223, BR352 and BR-365) of Triângulo Mineiro region and Alto Paranaíba, MG state. Continuous line = observed wealth richness (Mao Tau), dash-point line: estimated richness based on the second order Jacknife and dashed lines: estimated richness

based on Bootstrap.

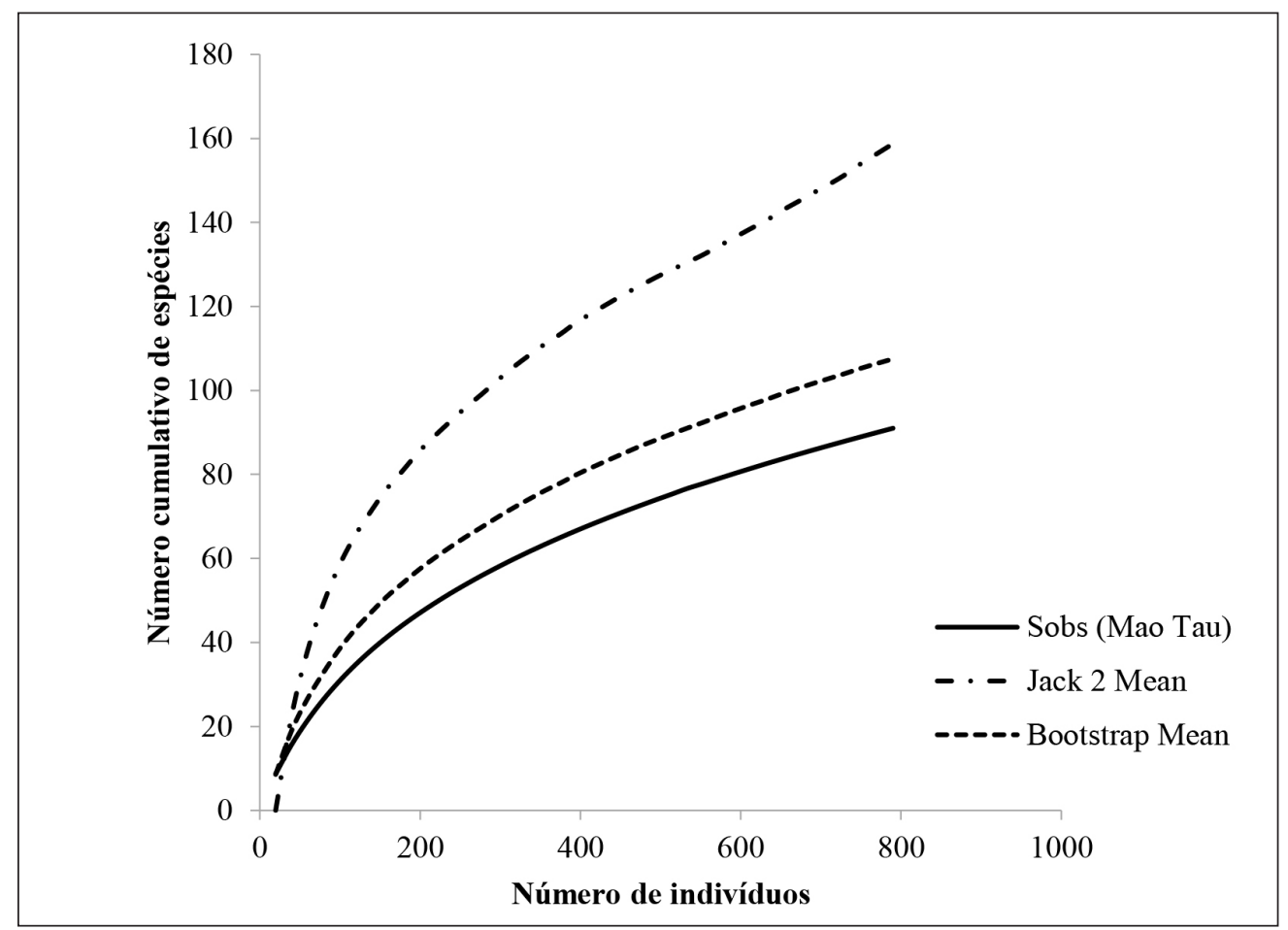

Fonte: Autores (2017)

Felfili e Felfili (2001) constataram que a distribuição da vegetação do Cerrado ocorre em mosaicos ao longo da paisagem com um grupo de espécies dominantes. Tal fato gera uma alta similaridade entre locais próximos, sendo necessário um elevado distanciamento ou diferenciações marcantes no terreno para que a similaridade entre as áreas seja reduzida. Portanto, o comportamento das curvas espécie-área deste trabalho deve ser devido à alta heterogeneidade dos ambientes de Cerrado amostrados, visto que o trabalho foi conduzido em uma grande área geográfica, com parcelas distantes entre si até 80 quilômetros, em diferentes altitudes e com características edáficas contrastantes, o que favoreceu o aparecimento de diferentes espécies ao longo do espaço-tempo, contribuindo para a não estabilização das curvas em nenhum dos três estimadores.

\section{Comparação com cerrados pouco impactados}

Ao se comparar este estudo com áreas de cerrado pouco antropizados (Tabela 2), é possível visualizar que a diversidade de Shannon $\left(3,64\right.$ nats.ind $\left.{ }^{-1}\right)$ está ligeiramente acima da média encontrada para os locais menos impactados. A riqueza se manteve próxima à média encontrada, apresentando 67 espécies por hectare em contraste com uma média de 68 espécies nos cerrados utilizados para comparação. Entretanto, a área basal encontrada foi ligeiramente menor que nas áreas preservadas e a 
densidade ficou muito abaixo, sendo inferior até ao menor desvio padrão calculado. Estes resultados são um indicativo de quão forte são os impactos recorrentes nas comunidades localizadas em beira de rodovias.

\section{Tabela 2 - Áreas de Cerrado preservado utilizadas para comparação estrutural com o presente trabalho. $\mathrm{Riq}=$ riqueza, $\mathrm{De}=$ densidade $\left(\mathbf{n} \cdot \mathrm{ha}^{-1}\right), \mathbf{A B}=$ área basal $\left(\mathrm{m}^{2} \cdot \mathrm{ha}^{-1}\right), \mathbf{H}^{\prime}=$ Índice de diversidade de Shannon.}

Table 2 - Preserved Cerrado areas used in the structural and diversity comparisons to the present study. $\mathrm{Riq}=$ richness, $\mathrm{De}=$ density $\left(\mathrm{n} \cdot \mathrm{ha}^{-1}\right), \mathrm{AB}=$ basal area $\left(\mathrm{m}^{2} \cdot \mathrm{ha}^{-1}\right), \mathrm{H}^{ }=$Shannon diversity index.

\begin{tabular}{|c|c|c|c|c|c|}
\hline Estado/áreas & Riq & De & $\mathrm{AB}$ & $\mathbf{H}^{\prime}$ & Referências \\
\hline APA do Paranoá - DF & 54 & 882 & 9,53 & 3,41 & Assunção e Felfili (2004) \\
\hline RECOR - IBGE - DF & 63 & 1964 & 12,28 & 3,53 & Andrade, Felfili e Violatti (2002) \\
\hline Brasília - DF & 53 & 1219 & 8,57 & 3,16 & Fonseca e Silva Júnior (2004) \\
\hline Brasília - DF & 54 & 970 & 6,67 & 3,40 & Fonseca e Silva Júnior (2004) \\
\hline Paraopeba - DF & 73 & 1990 & 18,13 & 3,57 & Balduino et al. (2005) \\
\hline Brasília - DF & 70 & 475 & - & 2,70 & Santana e Imaña-Encinas (2010) \\
\hline Caldas Novas - GO & 70 & 1626 & 12,81 & 3,26 & Lopes, Vale e Schiavini (2009) \\
\hline Caldas Novas - GO & 56 & 1907 & - & - & Silva et al. (2002) \\
\hline Alto Paraíso - GO & 92 & 944 & 8,05 & 3,46 & Felfili, Rezende e Silva Júnior (2007) \\
\hline Vila Propício - GO & 82 & 831 & 7,30 & 3,71 & Felfili, Rezende e Silva Júnior (2007) \\
\hline Serra Negra - GO & 97 & 1271 & 9,55 & 3,57 & Felfili, Rezende e Silva Júnior (2007) \\
\hline Serra Mesa-GO & 91 & 1019 & 9,17 & 3,57 & Felfili, Rezende e Silva Júnior (2007) \\
\hline Pirenópolis - GO & 56 & 507 & 3,91 & 3,33 & Moura et al. (2007) \\
\hline Chapada dos Veadeiros - GO & 85 & 1110 & 8,92 & 3,49 & Felfili, Rezende e Silva Júnior (2007) \\
\hline Uberlândia - MG & 76 & 1066 & 9,63 & 3,63 & Costa e Araújo (2001) \\
\hline Três Marias - MG & 81 & 2257 & 21,12 & 3,66 & Giácomo et al. (2013) \\
\hline Três Marias - MG & 57 & 926 & 6,73 & 3,43 & Giácomo et al. (2013) \\
\hline Buritis - MG & 90 & 796 & 11,87 & 3,75 & Nettesheim et al. (2010) \\
\hline Sete Lagoas - MG & 48 & 1408 & - & 3,09 & Pereira et al. (2013) \\
\hline Ribas do Rio Pardo - MS & 43 & 1052 & 5,81 & 3,23 & Carielo et al. (2012) \\
\hline Cuiabá - MT & 72 & 1358 & 13,25 & 3,34 & Oestreich Filho (2014) \\
\hline $\begin{array}{l}\text { Chapada dos Guimarães - } \\
\text { MT }\end{array}$ & 57 & 1178 & 13,70 & 3,56 & Oestreich Filho (2014) \\
\hline Água Boa - MT & 78 & 995 & 7,45 & 3,69 & Felfili et al. (2002) \\
\hline Patrocínio - SP & 53 & 1703 & - & 3,05 & Teixeira et al. (2004) \\
\hline Brotas - SP & 44 & 1150 & 7,20 & 3,02 & Durigan et al. (2002) \\
\hline Média & 68 & 1198 & 10,08 & 3,40 & \\
\hline Desvio Padrão & \pm 16 & \pm 468 & $\pm 4,10$ & $\pm 0,25$ & \\
\hline Beira de Rodovias - MG & 67 & 395 & 7,59 & 3,64 & Este trabalho \\
\hline
\end{tabular}

Fonte: Autores (2017) 
Porém, é importante ressaltar que apesar destes impactos antrópicos alterarem significativamente a densidade e a área basal da comunidade, os valores de riqueza e diversidade se mantiveram em níveis satisfatórios, com valores até superiores aos encontrados para as áreas preservadas. Isto reforça ainda mais a necessidade da criação de políticas públicas que venham contribuir na preservação, manutenção e recuperação destas comunidades, visto que o Cerrado, apesar de ser o segundo maior ecossistema do país em área e um dos hotspots mundiais para conservação da biodiversidade, não tem recebido destaque entre os programas governamentais de preservação, sendo muitas vezes ofuscado pela, não menos importante, elevada atenção dada aos impactos que ocorrem na Amazônia (FERNANDES; PESSÔA, 2011).

As correlações entre as variáveis estruturais (Figura 3) demonstram que a densidade e a área basal são positivamente correlacionadas (Figura 3B), assim como o número de espécies e o índice de diversidade de Shannon (Figura 3E). Assim, para os Cerrados SS, o aumento da densidade arbórea indica aumento na área basal e, por consequência, na biomassa aérea (RIBEIRO et al., 2011). A baixa densidade/área basal nas margens da rodovia denota perda da complexidade estrutural da fitofisionomia e provavelmente afeta a ocorrência de outras taxas nessas localidades. No entanto, a reduzida densidade/área basal não causou reflexo na riqueza e diversidade. A alta riqueza provavelmente se deve ao fato das parcelas terem sido alocadas distantes entre si, sendo amostradas diferentes áreas geográficas de cerrado, o que possibilita uma maior chance de se amostrar espécies novas, quando comparado com estudos nos quais se amostra uma única localização. Como a diversidade tende a ser fortemente dependente da riqueza (BATISTA et al., 2016), também esteve dentro dos padrões para cerrado. Assim, as margens da rodovia são, de fato, áreas que merecem maior atenção conservacionista por abrigarem espécies de cerrado em matrizes agropecuárias, todavia, novos estudos devem ser realizados para verificar quanto as populações de espécies de Cerrado estão sendo afetadas nestas localidades e até que ponto estas margens de rodovias podem se auto manter ao longo dos anos.

Figura 3 - Correlação entre variáveis estruturais e de diversidade de Cerrados sensu stricto amostrados no Brasil Central. Em cinza destacaram-se os cerrados localizados nas margens das rodovias.

Figure 3 - Correlation between structural and diversity variables of Cerrados sensu stricto sampled in central Brazil. In gray, we highlighted the Cerrados situated along the roadsides.

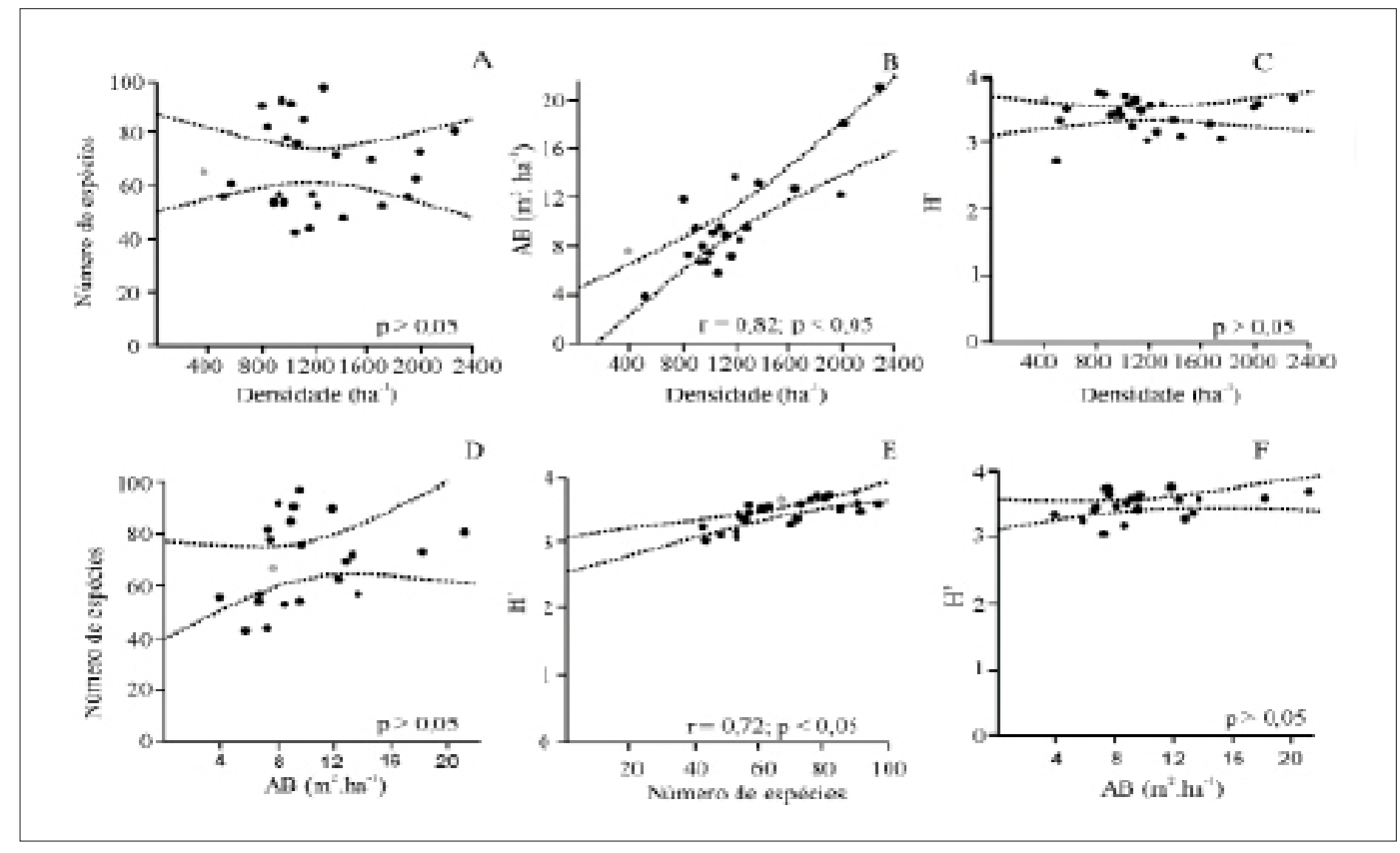

Fonte: Autores (2018) 


\section{Conclusão}

Foi possível com o estudo, concluir que a resiliência (capacidade de se recuperar após distúrbios) e a resistência (capacidade de absorver os distúrbios) das áreas de estudo foram alteradas. A paisagem se tornou rala e esparsa, fato comprovado pelos baixos valores de densidade e área basal, e, provavelmente, não voltará aos estágios anteriores sem o devido manejo ou proteção do fogo durante anos. Os impactos antrópicos recorrentes nessas áreas foram capazes de alterar significativamente a sua riqueza e estrutura, entretanto, notou-se que ainda assim, as mesmas conservam um montante significativo de espécies nativas, sendo assim, importantes aliadas na conservação do bioma Cerrado. Desta forma, torna-se necessária a criação de políticas públicas de cunho conservacionista. Desta forma, os resultados deste estudo poderiam ser aplicados em macroescala, visto que as margens de rodovias cortam todo o Bioma Cerrado, além de subsidiar novos e semelhantes estudos, abrangendo comunidades arbóreas, gramíneas e fauna.

\section{Agradecimentos}

Os autores agradecem à Fundação de Amparo à Pesquisa de Minas Gerais (FAPEMIG), Coordenação de Aperfeiçoamento de Pessoal de Nível Superior (CAPES) e Fundação de Amparo à Pesquisa do Estado de Goiás (FAPEG) pelas bolsas concedidas aos graduandos e mestrandos, à UEG pela Bolsa de Incentivo à Pesquisa (BIP) concedida ao coordenador do projeto e pelo financeiro através do Pró-Projetos/Pesquisa (Edital 29/2016).

\section{Referências}

ANDRADE, L. A. Z.; FELFILI, J. M.; VIOLATTI, L. Fitossociologia de uma área de Cerrado denso na RECOR-IBGE, Brasília-DF. Acta Botanica Brasilica, Belo Horizonte, v. 2, n. 16, p. 225-240, 03 abr. 2002.

ASSUNÇÃO, S. L.; FELFILI, J. M. Fitossociologia de um fragmento de cerrado sensu stricto na APA do Paranoá, DF, Brasil. Acta Botanica Brasilica, Belo Horizonte, v. 18, n. 4, p. 903-909, 2004.

BALDUINO, A. P. C. et al. Fitossociologia e análise comparativa da composição florística do cerrado da flora de Paraopeba-MG. Revista Árvore, Viçosa, v. 29, n. 1, p. 25-34, 2005.

BATISTA, A. P. B. et al. Species richness and diversity in shrub savanna using ordinary kriging. Pesquisa Agropecuária Brasileira, Brasília, v. 51, n. 8, p. 958-966, ago. 2016.

BYNG, J. W. et al. An update of the Angiosperm Phylogeny Group classification for the orders and families of flowering plants: APG IV. Botanical Journal of the Linnean Society, London, v. 181, n. 1, p. 1-20, 24 mar. 2016.

BOURSCHEID, K.; REIS, A. Dinâmica da invasão de Pinus elliottii Engelm. em restinga sob processo de restauração ambiental no Parque Florestal do Rio Vermelho, Florianópolis, SC. Biotemas, Florianópolis, v. 23, n. 2, p. 23-30, 8 abr. 2011.

BRASIL. Ministério do Meio Ambiente. Áreas prioritárias para conservação, uso sustentável e repartição de benefícios da biodiversidade brasileira. Atualização: Portaria MMA no 9, de 23 de janeiro de 2007. Brasília: MMA, 2007.

BRASIL. Ministério do Meio Ambiente. Plano de ação para prevenção e controle do desmatamento e das queimadas: Cerrado. Brasília: MMA, 2011. 200 p.

BRAZ, V. S.; KANEGAE, M. F.; FRANCO, A. C. Estabelecimento e desenvolvimento de Dalbergia miscolobium Benth. em duas fitofisionomias típicas dos Cerrados do Brasil 
Central. Acta Botanica Brasilica, Belo Horizonte, v. 14, n. 1, p. 27-35, 2000.

BROWER, J. E.; ZAR, J. H. Field and laboratory methods for general ecology. Dubuque: Wm. C. Brown Company, 1984. 226 p.

CARIELO, P. et al. Levantamentos florístico e fitossociológico de um trecho de cerrado da Fazenda Boa Aguada, município de Ribas do Rio Pardo - MS. Colloquium Agrariae, Presidente Prudente, v. 8, nesp, p. 63-70, 2012.

COLWELL, R. K. EstimateS: Statistical estimation of species richness and shared species form samples. Versão 8.2. [S.l.: s.n.], 2005.

COSTA, A. A.; ARAÚJO, G. M. Comparação da vegetação arbórea de cerradão e cerrado na Reserva do Panga, Uberlândia, Minas Gerais. Acta Botanica Brasilica, Belo Horizonte, v. 15, n. 1, p. 63-72, 2001.

DURIGAN, G. et al. Caracterização de dois estratos da vegetação em uma área de cerrado no município de Brotas, SP, Brasil. Acta Botanica Brasilica, Belo Horizonte, v. 16, n. 3, p. 251-262, 2002.

FALLEIROS, R. M.; ZENNI, R. D.; ZILLER, S. R. Invasão e manejo de Pinus taeda em campos de altitude do Parque Estadual do Pico Paraná, Paraná, Brasil. Floresta, Curitiba, v. 41, n. 1, p. 123-134, 2011.

FELFILI, M. C.; FELFILI, J. M. Diversidade alfa e beta no cerrado sensu stricto da Chapada Pratinha, Brasil. Acta Botanica Brasilica, Belo Horizonte, v. 15, n. 2, p. 243-254, ago. 2001.

FELFILI, J. M. et al. Composição florística e fitossociologia do cerrado sentido restrito no município de Água Boa - MT. Acta Botanica Brasilica, Belo Horizonte, v. 16, n. 1, p. 103-112, 2002.

FELFILI, J. M.; CARVALHO, F. A.; HAIDAR, R. F. Manual para o monitoramento de parcelas permanentes nos biomas Cerrado e Pantanal. Brasília: UNB, 2005. 54 p.

FELFili, J. M.; REZENDE, A. V.; SILVA JÚNIOR, M. C. Biogeografia do Bioma Cerrado: vegetação e solos da Chapada dos Veadeiros. Brasília: UnB, 2007. 256 p.

FERNANDES, P. A.; PESSÔA, V. L. S. O Cerrado e suas atividades impactantes: uma leitura sobre o garimpo, a mineração e a agricultura mecanizada. Observatorium: Revista Eletrônica de Geografia, Uberlândia, v. 3, n. 7, p. 19-37, out. 2011.

FIEDLER, N. C. et al. Efeito de incêndios florestais na estrutura e composição florística de uma área de cerrado sensu stricto na fazenda Água Limpa-DF. Revista Árvore, Viçosa, MG, v. 28, n. 1, p. 129-138, fev. 2004.

FONSECA, M. S.; SILVA JÚNIOR, M. C. Fitossociologia e similaridade florística entre trechos de cerrado sentido restrito em interflúvio e em vale no Jardim Botânico de Brasília, DF. Acta Botanica Brasilica, Belo Horizonte, v. 18, n. 1, p. 19-29, 2004.

FORZZA, R. C. et al. New Brazilian floristic list highlights conservation challenges. Bioscience, Oxford, v. 62, n. 1, p. 39-45, jan. 2012.

FRANÇOSO, R. D.; HAIDAR, R. F.; MACHADO, R. B. Tree species of South America central savanna: endemism, marginal areas and the relationship with other biomes. Acta Botanica Brasilica, Belo Horizonte, v. 30, n. 1, p. 78-86, mar. 2016.

FRANZON, R. C. et al. Araçás do Gênero Psidium: principais espécies, ocorrência, descrição e usos. Planaltina: Embrapa, 2009. 47 p.

GIÁCOMO, R. G. et al. Florística e fitossociologia em áreas de campo sujo e cerrado sensu 
stricto na estação ecológica de Pirapitinga - MG. Ciência Florestal, Santa Maria, v. 23, n. 1, p. 29-43, 2013.

GIRÃO, L. C. et al. Changes in tree reproductive traits reduce functional diversity in a fragmented atlantic forest landscape. Plos One, San Francisco, v. 2, n. 9, p. 1-12, set. 2007.

GOOGLE. Google Earth. Version 7.1.8.3036 (32-bit). 2017. Nota (Localização geográfica das parcelas na região do estudo). Disponível em: <https://www.google.com.br/earth/download/ge/ agree.html>. Acesso em: 21 mar. 2017.

IBGE. Manual Técnico da Vegetação Brasileira. Rio de Janeiro: Ministério do Planejamento, Orçamento e Gestão; Instituto Brasileiro de Geografia e Estatística, 2012. 271 p.

KAGEYAMA, P. Y.; GANDARA, F. B.; SOUZA, L. M. I. Consequências genéticas da fragmentação sobre populações de espécies arbóreas. Série Técnica IPEF, Piracicaba, v. 12, n. 32, p. 65-70, dez. 1998.

KLINK, C. A.; MACHADO, R. B. A conservação do Cerrado brasileiro. Megadiversidade, Rio de Janeiro, v. 1, n. 1, p. 147-155, jul. 2005.

KOTTEK, M. et al. World Map of the Köppen-Geiger climate classification updated. Meteorologische Zeitschrift, Stuttgart, v. 15, n. 3, p. 259-263, jun. 2006.

KREBS, C. J. Ecological methodology. New York: Wesley Longman, 2000.

LAURANCE, W. F; VASCONCELOS, H. L. Consequências ecológicas da fragmentação florestal na Amazônia. Oecologia Brasiliensis, Rio de Janeiro, v. 13, n. 3, p. 434-451, set. 2009.

LOPES, S. F.; VALE, V. S.; SCHIAVINI, I. Efeito de queimadas sobre a estrutura e composição da comunidade vegetal lenhosa do cerrado sentido restrito em Caldas Novas, GO. Revista Árvore, Viçosa, MG, v. 33, n. 4, p. 695-704, ago. 2009.

MACHADO, Ricardo B. et al. Estimativas de perda da área do Cerrado brasileiro. Brasília: Conservation International do Brasil, 2004.

MASSI, K. G. Efeitos da adição de nutrientes em espécies arbóreas e herbáceas de Cerrado. 2014. 109 f. Tese (Doutorado) - Universidade de Brasília, Brasília, 2014.

MATTEUCCI, S. D.; COLMA, A. Metodologia para el estudio de la vegetacion. Coro: Secretaría General de La Organización de Los Estados Americanos, 1982. 86 p.

MENDES, M. Por que é importante investir em infraestrutura? 2011. Disponível em: $<$ http://www.brasil-economia-governo.org.br/2011/02/09/por-que-e-importante-investir-eminfraestrutura/>. Acesso em: 11 maio 2016.

MIASHIKE, R. L. Invasão por Pinus spp. em fisionomias campestres do Cerrado, no estado de São Paulo. 2015. 101 f. Dissertação (Mestrado em Ecologia) - Universidade de São Paulo, São Paulo, 2015.

MITTERMEIER, R. A. et al. Hotspots revisited: earth's biologically richest and most endangered terrestrial ecoregions. Mexico: CEMEX; Agrupación Sierra Madre, 2004. 392 p.

MOURA, I. O. et al. Fitossociologia da comunidade lenhosa de uma área de cerrado rupestre no Parque Estadual dos Pireneus, Pirenópolis, Goiás. Revista de Biologia Neotropical, Goiânia, v. 4, n. 2, p. 83-100, 2007.

NETTESHEIM, F. C. et al. Estrutura e florística do estrato arbóreo no cerrado sensu stricto de Buritis, Minas Gerais, Brasil. Rodriguésia, Rio de Janeiro, v. 61, n. 4, p. 731-747, 2010.

OESTREICH FILHO, E. Fitossociologia, diversidade e similaridade entre fragmentos de cerrado stricto sensu sobre neossolos quartzarênicos órticos, nos municípios de Cuiabá e 
Chapada dos Guimarães, estado de Mato Grosso, Brasil. 2014. 88 f. Dissertação (Mestrado) Universidade Federal de Mato Grosso, Cuiabá, 2014.

PAIVA, R. et al. (Ed.). CAGAITEIRA (Eugenia dysenterica DC.). Lavras: UFLA, 2009. 21 p.

PEREIRA, I. M. et al. Estrutura fitossociológica de uma área de cerrado sensu stricto em Sete Lagoas, MG. Enciclopédia Biosfera, Goiânia, v. 9, n. 17, p. 3433-3446, 2013.

POTT, A.; POTT, V. J. Plantas nativas para recuperação de áreas degradadas e reposição da vegetação no Mato Grosso do Sul. Campo Grande: EMBRAPA gado de corte, 2002. (Comunicado Técnico EMBRAPA, n. 75).

RAD, J. E.; MANTHEY, M.; MATAJI, A. Comparison of plant species diversity with different plant communities in deciduous forests. International Journal of Environmental Science \& Technology, [S.1.], v. 6, n. 3, p. 389-394, 2009.

RATTER, J. A.; BRIDGEWATER, S.; RIBEIRO, J. F. Analysis of the floristic composition of the brazilian Cerrado vegetation III: Comparison of the woody vegetation of 376 areas. Edinburgh Journal of Botany, Cambridge, v. 60, n. 1, p. 57-109, 2003.

RIBEIRO, S. C. et al. Above and belowground biomass in a Brazilian Cerrado. Forest Ecology and Management, Amsterdam, v. 262, n. 3, p. 491-499, ago. 2011.

RIBEIRO, J. F.; WALTER, B. M. T. As principais fitofisionomias do bioma Cerrado. In: SANO, S. M.; ALMEIDA, S. P.; RIBEIRO, J. F. (Ed.). Cerrado: ecologia e flora. Brasília: EMBRAPA, 2008. p. 151-199.

ROSA, P. A. L. et al. Recuperação de um solo de cerrado após 19 anos: ocorrência espontânea de espécies arbóreas. Revista de Agricultura Neotropical, Cassilândia, v. 1, n. 1, p. 44-57, jul./set. 2014.

SAMPAIO, R. S.; BRITO, P. C. R. Impactos ambientais causados pela construção de rodovias. 2009. Tese (Doutorado) - Universidade Católica do Salvador, Salvador, 2009.

SANTANA, O. A.; IMAÑA-ENCINAS, J. Fitossociologia das espécies arbóreas nativas de cerrado em áreas adjacentes a depósitos de resíduos domiciliares. Floresta, Curitiba, v. 40, n. 1, p. 93-110, 2010.

SARTORELLI, P. A. R. et al. Rebrota após fogo de espécies arbóreas de diferentes grupos fenológicos foliares em Cerrado Stricto Sensu. Revista Científica Eletrônica de Engenharia Florestal, Garça, v. esp., n. 10, p. 1-13, ago. 2007.

SCHILLING, A. C.; BATISTA, J. L. F. Curva de acumulação de espécies e suficiência amostral em florestas tropicais. Revista Brasileira de Botânica, São Paulo, v. 31, n. 1, p. 179-187, mar. 2008.

SILVA, L. O. et al. Levantamento florístico e fitossociológico em duas áreas de cerrado sensu stricto no Parque Estadual da Serra de Caldas Novas, Goiás. Acta Botanica Brasilica, Belo Horizonte, v. 16, n. 1, p. 43-53, 2002.

SILVEIRA, E. P.; COSTA, R. B.; FELFILI, J. M. Florística da vegetação remanescente de Cerrado sensu stricto em terra indígena no noroeste de Mato Grosso, Brasil. Revista de Biologia Neotropical, Goiânia, v. 6, n. 2, p. 15-25, 2009.

TEIXEIRA, M. I. J. G. et al. Florística e fitossociologia de área de Cerrado S.S. no município de Patrocínio Paulista, nordeste do estado de São Paulo. Bragantia, Campinas, v. 63, n. 1, p. 1-11, 2004.

TROPICOS.ORG. Missouri Botanical Garden. Saint Louis: Missouri Botanical Garden, 2016. Disponível em: <http://www.tropicos.org>. 
VASCONCELOS, P. B. O papel das beiras de estradas na conservação da diversidade vegetal do Cerrado. 2012. 41 f. Dissertação (Mestrado) - Universidade Federal de Uberlândia, Uberlândia, 2012.

VASCONCELOS, P. B.; ARAÚJO, G. M.; BRUNA, E. M. The role of roadsides in conserving Cerrado plant diversity. Biodiversity and Conservation, [S.1.], v. 23, n. 12, p. 3035-3050, 2014. 